\title{
Enhanced localization of liposomes with prolonged blood circulation time in infected lung tissue
}

\author{
Irma A.J.M. Bakker-Woudenberg ', August F. Lokerse ', Marian T. ten Kate ${ }^{1}$ \\ and Gert Storm ${ }^{2}$ \\ 'Department of Clinical Microbiology, Erasmus Unicersity, Rotterdam (The' Netherlands) and '2 Department of Pharmaceutics, \\ University of Utrecht, Utrecht (The Netherlands)
}

(Received 28 November 1991)

Key words: Liposome; Tissue distribution; Lung infection

In an experimental model of unilateral pneumonia caused by Klebsiella pneumoniae in rats we investigated whether intravenous administration of liposomes with prolonged blood circulation time resulted in significant localization of liposomes in infected lung tissue. Liposomes $(100 \mathrm{~nm})$ composed of hydrogenated phosphatidylinositol: hydrogenated phosphatidylcholine : cholesterol (molar ratio, 1:10:5) radiolabeled with gallium-67-deferoxamine showed relatively long blood circulation time. The degree of localization of these long circulating liposomes in the infected left lung was significantly higher compared to that of localization of $110 \mathrm{~nm}$ egg phosphatidylglycerol : egg phosphatidylcholine : cholesterol (molar ratio, 1:10:5) liposomes which exhibited relatively short blood circulation time. At $16 \mathrm{~h}$ after administration of the long circulating liposomes (when $10 \%$ of the injected dose was still present in the bloodstream) localization of liposomes in the infected left lung was increased up to 10-fold compared to the left lung of uninfected rats, and appeared to be highly correlated with the intensity of the infection. In the uninfected right lung the localization of long circulating liposomes was not increased. The degree of localization of liposomes in the infected tissue is dependent on the residence time of liposomes in the blood compartment. The extent of localization of long circulating liposomes in infected tissue appeared to be dependent on the liposomal dose administered.

\section{Introduction}

It is well-known from clinical experience that, despite the availability of potent antibiotics, infections are still a major cause of morbidity and mortality. There is an evident therapeutic need for improvement of antibiotic treatment which should result in increased antibiotic concentrations in infected tissues or in infected cells, and in reduced toxicity of potentially toxic antibiotics. Application of liposomes as carriers of antimicrobial agents has been proposed as a potential way to increase the selective delivery of the antimicrobial agents to their target sites. Unfortunately, some

\footnotetext{
Abbreviations: HPI, hydrogenated soybean phosphatidylinositol; HPC, hydrogenated soybean phosphatidylcholine; PG, egg phosphatidylglycerol; PC, egg phosphatidylcholine; DF, deferoxamine mesylate.

Correspondence: I.A.J.M. Bakker-Woudenberg, Department of Clinical Microbiology, Erasmus University, P.O. Box 1738, 3000 DR Rotterdam, The Netherlands.
}

major problems have not yet been solved. Directing liposomes to infected tissues and cells other than liver and spleen is hampered by preferential uptake of intravenously administered liposomes by cells of the mononuclear phagocyte system (MPS) particularly those residing in liver and spleen. Accordingly, it is not surprising that most experimental data on the use of liposomes as carriers of antibiotics are derived from animal models of intracellular infections involving the MPS [1-7]. In these studies an improved therapeutic effect and/or reduced toxicity resulting from encapsulation of the antibiotics in liposomes have been demonstrated. Effective targeting of liposomes to non-MPS tissues would require avoidance of uptake by the MPS. Recent work has shown that specific changes in the liposome composition can drastically reduce the rate and extent of uptake by the liver and spleen. Such liposomes can circulate in the blood for prolonged periods of time, when compared to 'conventional' liposomes with lipid compositions usually used. Studies of Gabizon et al. and Allen et al. show important progress in this respect [8-12]. They evaluated the biodistribu- 
tion of a series of liposome formulations differing in mean particle size and lipid composition. They found that specific liposome formulations with prolonged blood circulation time showed enhanced localization in areas of implanted tumors in mice. In this study it was investigated whether infected tissue is more accessible to long circulating liposomes than to conventional (short circulating) liposomes. In addition, it was studied whether the degree of localization in infected tissue is dependent on the intensity of infection. A well established model of pneumonia caused by Klebsiella pneumoniae in rats was used for the present investigations.

\section{Materials and Methods}

Hydrogenated soybean phosphatidylinositol (HPI), hydrogenated soybean phosphatidylcholine (HPC), egg phosphatidylglycerol (PG) and egg phosphatidylcholine (PC) were obtained from Avanti Polar Lipids (Alabaster, AL). Cholesterol was from Sigma (St. Louis, MO). ${ }^{67} \mathrm{Ga}$-citrate and ${ }^{111} \mathrm{In}$-chloride were from Frosst (Quebec, Canada). Deferoxamine mesylate (DF) was from Ciba-Geigy (Basel, Switzerland). The acetate form of AG1X2 resin (acetate form, 200-400 mesh) was from Bio-Rad (Richmond, CA).

Liposomes. Liposome preparations consisted of either HPI:HPC: Chol in a molar ratio $1: 10: 5$ or PG : PC: Chol in a molar ratio $1: 10: 5$. Liposomes were prepared as described previously [13] with some modifications. In brief, a chloroform/methanol $(9: 1, \mathrm{v} / \mathrm{v})$ solution of the lipid mixture was evaporated to dryness in a round-bottomed flask. The lipids were redissolved in $2 \mathrm{ml}$ of cyclohexane and were lyophilized. Multilamellar vesicles were formed by Vortex mixing of the lipid film in a buffer solution containing $10 \mathrm{mM}$ Hepes, $150 \mathrm{mM} \mathrm{NaCl}$ and $25 \mathrm{mM}$ DF (pH 7.4). Lipid concentration was $100 \mu \mathrm{mol} / \mathrm{ml}$. Vortex mixing was performed for 1220 -s periods at room temperature or, in the case of HPI : HPC: Chol liposomes at $62^{\circ} \mathrm{C}$ because of the high transition temperature of the phospholipids used. After Vortex mixing HPI : HPC: Chol liposomes were incubated for $1 \mathrm{~h}$ at $62^{\circ} \mathrm{C}$. Liposomes were extruded 10 times through polycarbonate filters of 0.05 $\mu \mathrm{m}$ pore size (Nuclepore; Pleasanton, CA), using an extruder device from Lipex Biomembranes (Vancouver, Canada). For sizing of the HPI: HPC: Chol liposomes the extruder device was preheated to $62^{\circ} \mathrm{C}$. Liposomes were separated from non-entrapped DF by gel filtration on a Sephadex G-50 columm (Pharmacia, Uppsala, Sweden) with $10 \mathrm{mM}$ Hepes and $150 \mathrm{mM} \mathrm{NaCl}$ ( $\mathrm{pH}$ 7.4) as the elution buffer. Phospholipid concentration was determined by a phosphate assay [14]. Mean particle size was determined by dynamic light scattering (Malvern 4700 system, Malvern, U.K.). As a measure of the particle size distribution of the dispersion the system reports a polydispersity index. This index ranges from 0.0 for an entirely monodisperse up to 1.0 for a completely polydisperse system. The mean particle size of the liposomes used was $100 \pm 4 \mathrm{~nm}$ with a polydispersity index of $0.13 \pm 0.02$ (mean \pm S.D. of 9 preparations) for the HPI:HPC: Chol liposomes, and $110 \pm 9 \mathrm{~nm}$ with a polydispersity index of $0.21 \pm 0.07$ (mean \pm S.D. of 3 preparations) for the PG: $\mathrm{PC}$ : Chol liposomes. Liposomes were stored at $4^{\circ} \mathrm{C}$ under nitrogen for at most $24 \mathrm{~h}$.

Radiolabeling of liposomes. The liposomes were radiolabeled with ${ }^{67} \mathrm{Ga}$-deferoxamine $\left({ }^{67} \mathrm{Ga}\right.$-DF $)$ as described by Gabizon et al. [15]. The labeling resulted in formation of ${ }^{67} \mathrm{Ga}-\mathrm{DF}$ complex in the aqueous interior of liposomes. Non-entrapped ${ }^{67} \mathrm{Ga}$-oxine was removed by passing the liposome suspension through an anionexchange resin (AG1X2).

Animals and Infection. Female $\mathrm{R}$ strain albino-rats (specific pathogen free; 14 to 18 weeks old; weight, 185 to $215 \mathrm{~g}$; bred at ITRI-TNO, Rijswijk, The Netherlands) were used in all experiments. Experimental infection was induced using a Klebsiella pneumoniae strain (ATCC 43816, capsular serotype 2). Cultures were obtained by incubation for $16 \mathrm{~h}$ at $37^{\circ} \mathrm{C}$ in IsoSensitest broth (Oxoid, London, U.K.). After proper dilution and reincubation for $2 \mathrm{~h}$ at $37^{\circ} \mathrm{C}$ suspensions of logarithmically growing bacteria were prepared. A unilateral pneumonia (left lung) was produced as described previously [16]. In brief, rats were anesthetized with fluanisone (Hypnorm $^{\mathrm{R}}$; Duphar B.V., Amsterdam, The Netherlands) and pentobarbital (Abbott Laboratories, North Chicago, IL). The left main stem bronchus was intubated and the left lung was inoculated with $0.02 \mathrm{ml}$ of saline suspension containing $10^{5} \mathrm{cfu}$ of $K$. pneumoniae. After bacterial inoculation the narcotic antagonist Nalorphine bromide (Onderlinge Pharmaceutische Groothandel, Utrecht, The Netherlands) was injected. The severity of the resulting infection in the lung was assessed by measuring the increase in bacterial numbers as well as increase in weight of the infected lung tissue. Numbers of bacteria in the left lung and right lung were quantitated as follows. Animals were killed, the left lung and the right lung were removed separately, weighed and homogenized in 20 $\mathrm{ml}$ of phosphate-buffered saline (PBS) for 30-s at 10000 rpm in a VirTis homogenizer (VirTis, Gardiner, NY). Serial 10 -fold dilutions of homogenates were prepared. Vols. of $0.2 \mathrm{ml}$ of each dilution as well as $1 \mathrm{ml}$ vols. of the undiluted lung homogenate were spread on tryptone soy agar (Mast International, Merseyside, U.K.).

The histological features of the pneumonic lesions were studied after fixation of the lungs by injecting the trachea with $10 \%$ formalin under constant pressure to reexpand the lungs. Segments of the left lung were then dehydrated in ethanol and toluol, embedded in paraffin, sectioned and stained with hematoxylin-eosin or by the Gram stain technique. 
Biodistribution studies. In uninfected and infected rats, liposome blood circulation time and localization of liposomes in liver, spleen, kidney, left lung and right lung was determined using the high affinity ${ }^{67} \mathrm{Ga}-\mathrm{DF}$ complex as a liposome marker. Correction for the blood content of the tissues was performed using ${ }^{111}$ In-oxine-labeled syngeneic erythrocytes injected intravenously $10 \mathrm{~min}$ before dissection. Labeling of erythrocytes was performed as described by Heaton et al. [17]. Liposomes were injected intravenously in a vol. of $0.9 \mathrm{ml}$ at doses of $5,10,15,20$ or $25 \mu \mathrm{mol}$ liposomal lipid per rat $(200 \mathrm{~g})$. At different time intervals after injection of liposomes blood samples of $1 \mathrm{ml}$ were obtained by retroorbital bleeding under $\mathrm{CO}_{2}$ anesthesia. Then the rats were killed, and the spleen, kidney, left lung (one lobe), right lung (four lobes) and liver were removed. Radioactivity was quantitated in a $\gamma$ counter (Minaxy 5530, Packard Instruments, Downers Grove, U.S.A.). Encapsulated ${ }^{67} \mathrm{Ga}$ served as a marker to determine the distribution of intact liposomes in the various tissues.

Statistical analysis. Differences in blood circulation time and localization in liver and spleen between HPI:HPC: Chol liposomes and PG:PC:Chol liposomes were statistically evaluated by using the Wilcoxon signed rank test (two tailed). Student's $t$ test was used for evaluation of differences in blood level and hepatosplenic uptake of HPI:HPC:Chol liposomes between infected rats and uninfected rats. The Spearman rank test was used to calculate whether the localization of HPI:HPC: Chol liposomes or PG: PC: Chol liposomes in infected left lung was correlated with the increase in weight of the left lung. In addition, this test was used to detect whether there was a relationship between left lung localization and injected liposomal lipid dose.

\section{Results}

Biodistribution of HPI:HPC: Chol liposomes and $P G: P C:$ Chol liposomes in uninfected rats

Both types of liposomes under investigation (HPI:HPC: Chol and PG:PC: Chol liposomes) had a similar mean diameter of about $100 \mathrm{~nm}$. The liposome blood circulation time and localization of liposomes in liver, spleen, kidney and lung after i.v. administration (15 $\mu \mathrm{mol}$ liposomal lipid per rat) are shown for both types of liposomes in Table I. The HPI: HPC: Chol liposomes were eliminated from the bloodstream at a much lower rate than the PG:PC:Chol liposomes $(P=0.0001)$. At $10 \mathrm{~h}$ after administration, $23.4 \pm 0.6 \%$ (mean \pm S.E.) of the administered dose of HPI : HPC: Chol liposomes was still present in the circulation compared to $2.9 \pm 0.3 \%$ in the case of $P G: P C:$ Chol liposomes. The relatively long blood circulation time of the HPI:HPC:Chol liposomes was accompanied by initially low uptake of liposomes by liver and spleen (at $0.5 \mathrm{~h}$ after administration being 4.8 and $0.2 \%$, respectively), which gradually increased with time (up to a maximum of 25.8 and $1.2 \%$, respectively, at $16 \mathrm{~h}$ after liposome administration). At $0.5 \mathrm{~h}$ after administration total recovery of liposomes based on blood, liver, spleen, kidney and lung uptake was $93 \%$. The initial uptake of PG: PC: Chol liposomes by liver and spleen at $0.5 \mathrm{~h}$ after administration was significantly increased

\section{TABLE I}

Biodistribution of ${ }^{67} \mathrm{Ga}-\mathrm{DF}$ labeled liposomes in uninfected rats at larious time intertals after injection

Liposomes composed of HPI:HPC: Chol (molar ratio, 1:10:5) or PG:PC:Chol (molar ratio, 1:10:5) were injected intravenously at a dose of 15 $\mu$ mol liposomal lipid per rat. Data are expressed as mean \pm S.E. of four rats.

\begin{tabular}{|c|c|c|c|c|c|}
\hline \multirow{2}{*}{$\begin{array}{l}\text { Liposome } \\
\text { composition }\end{array}$} & \multicolumn{5}{|c|}{ Tissue radioactivity (\% of injected dose) } \\
\hline & $0.5 \mathrm{~h}$ & $5 \mathrm{~h}$ & $10 \mathrm{~h}$ & $16 \mathrm{~h}$ & $24 \mathrm{~h}$ \\
\hline \multicolumn{6}{|c|}{ HPI:HPC:Chol } \\
\hline blood & $81.1 \pm 4.3$ & $61.6 \pm 2.5$ & $23.4 \pm 0.6$ & $10.2 \pm 0.5$ & $4.0 \pm 0.7$ \\
\hline liver & $4.8 \pm 0.8$ & $10.4 \pm 1.7$ & $22.2 \pm 1.5$ & $25.8 \pm 0.6$ & $22.8 \pm 1.4$ \\
\hline spleen & $0.2 \pm 0.1$ & $0.6 \pm 0.2$ & $1.4 \pm 0.3$ & $1.2 \pm 0.2$ & $0.8+0.1$ \\
\hline kidney & $6.2 \pm 0.7$ & $0.8 \pm 0.1$ & $1.0 \pm 0.1$ & $1.3 \pm 0.1$ & $0.9 \pm 0.2$ \\
\hline lung & $0.5 \pm 0.3$ & $0.04 \pm 0.0$ & $0.2 \pm 0.0$ & $0.1 \pm 0.0$ & $0.1 \pm 0.0$ \\
\hline recovery & $92.8 \pm 3.3$ & $73.7 \pm 1.4$ & $48.2 \pm 1.5$ & $38.5 \pm 0.6$ & $28.5+1.5$ \\
\hline \multicolumn{6}{|l|}{ PG : PC: Chol } \\
\hline blood & $41.9 \pm 1.3$ & $11.7 \pm 1.2$ & $2.9 \pm 0.3$ & $1.8 \pm 0.1$ & $0.7 \pm 0.2$ \\
\hline liver & $28.4 \pm 1.3$ & $16.2 \pm 0.7$ & $10.1 \pm 0.6$ & $9.6 \pm 0.3$ & $9.0 \pm 0.2$ \\
\hline spleen & $2.3 \pm 0.5$ & $4.3 \pm 0.3$ & $2.3 \pm 0.2$ & $2.3 \pm 0.1$ & $2.4 \pm 0.4$ \\
\hline kidney & $2.9 \pm 0.6$ & $1.8 \pm 0.2$ & $1.1 \pm 0.1$ & $0.6 \pm 0.1$ & $0.9 \pm 0.1$ \\
\hline lung & $1.2 \pm 0.1$ & $0.8 \pm 0.1$ & $0.3 \pm 0.1$ & $0.1 \pm 0.0$ & $0.1 \pm 0.0$ \\
\hline recovery & $76.6 \pm 1.9$ & $34.9 \pm 1.3$ & $16.7 \pm 0.4$ & $14.4 \pm 0.1$ & $13.1+0.7$ \\
\hline
\end{tabular}


( $P=0.03)$ compared to HPI : HPC: Chol liposomes being $28.4 \%$ and $2.3 \%$, respectively. Concomitantly the blood circulation time of PG : PC: Chol liposomes was relatively short and significantly reduced $(P=0.0001)$ compared to that of the HPI:HPC: Chol liposomes. Total recovery of PG:PC: Chol liposomes based on blood, liver, spleen, kidney and lung uptake at $0.5 \mathrm{~h}$ after administration was $77 \%$.

\section{Course of K. pneumoniae lung infection}

After inoculation of the left lung with $10^{5} \mathrm{cfu}$ of $K$. pneumoniae a progressive development of the infection started. Within 3-4 days bacterial numbers in the left lung increased up to $2 \cdot 10^{10}$. The involvement of the left lung tissue in the infectious process was clearly demonstrated (Fig. 1). Substantial bacterial increase was associated with an increase in severity of infection reflected by an increase in weight of the infected left lung from 0.5 up to $2 \mathrm{~g}$. (Macroscopic examination revealed that large parts of the left lung were involved in the infectious process.) Histological examination revealed that the pneumonic lesions were characterized by edema fluid, large numbers of Gram-negative bacilli and a cellular infiltrate composed of polymorphonuclear leukocytes and a few macrophages. Within 3-4 days all rats had bacteria in the blood, resulting in the appearance of bacteria in other tissues such as the right lung in which bacterial numbers ranged from 10 up to $10^{9}$ (Fig. 1). This, however, was not associated with development of an infectious process as reflected by the absence of increase in weight of the right lung and confirmed by histological examination.

\section{Course of infection}
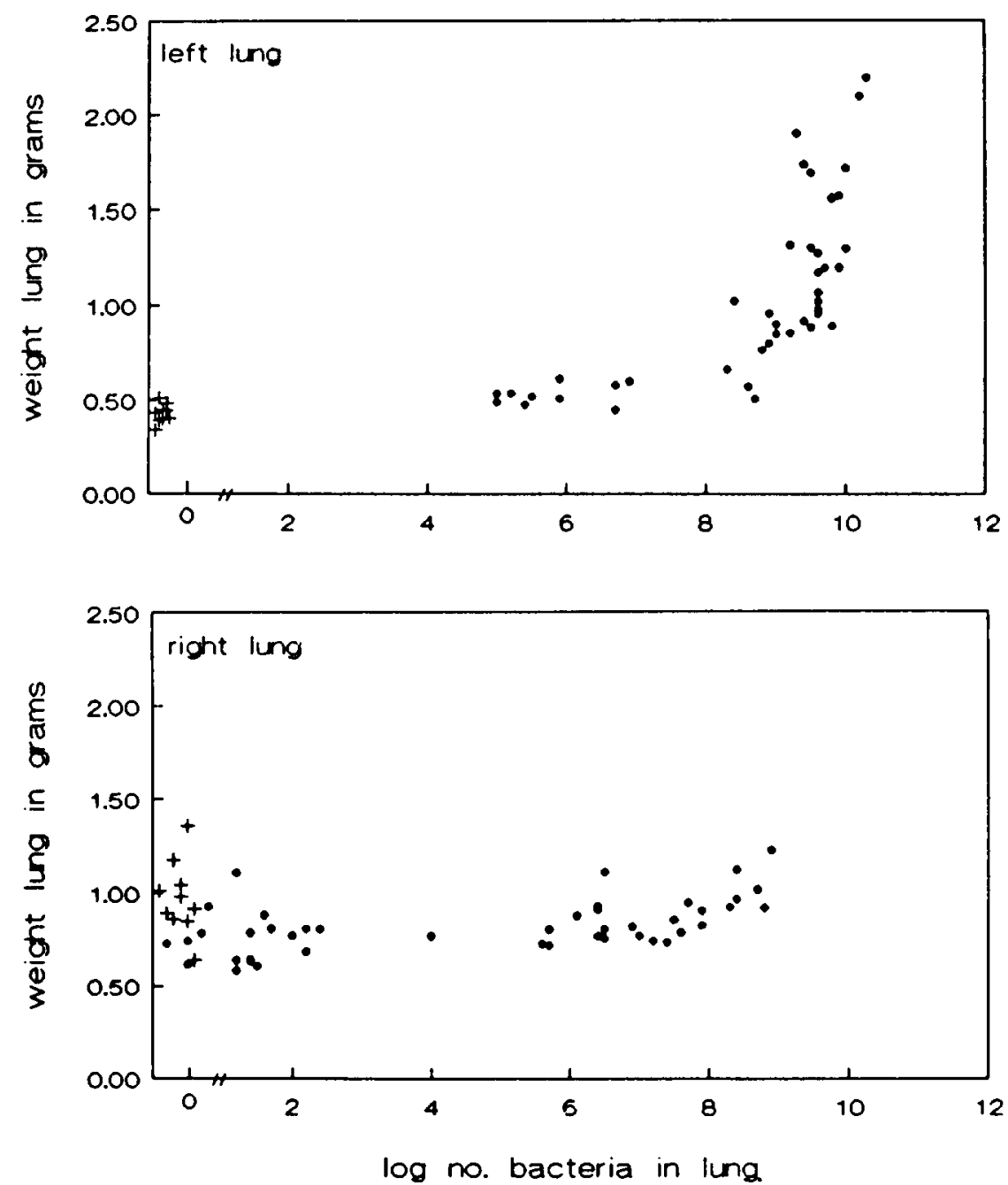

Fig. 1. Course of Klebsiella pneumoniae lung infection after inoculation of the left lung with $10^{5}$ cfu of Klebsiella pneumoniae. Increase in bacterial numbers and increase in weight of infected lung tissue was determined in a group of 44 infected rats (•). Weight of uninfected lung tissue was determined in a group of 10 uninfected rats $(+)$. 


\section{Biodistribution HPI:HPC:Chol liposomes \\ infected rats vs. uninfected rats}

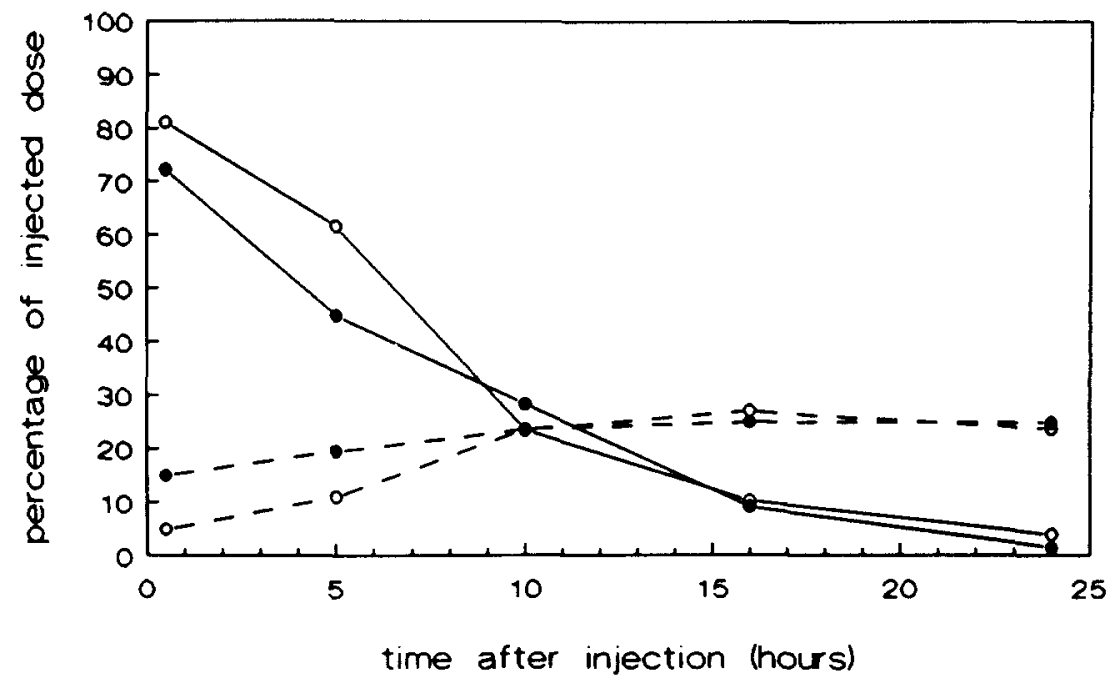

Fig. 2. Biodistribution of ${ }^{67} \mathrm{Ga}-\mathrm{DF}$ labeled HPI : HPC: Chol (molar ratio, $1: 10: 5$ ) liposomes in rats at various intervals after injection. A dose of $15 \mu \mathrm{mol}$ liposomal lipid per rat was injected intravenously in rats with Klebsiella pneumoniae lung infection $(\bullet)$ or in uninfected rats $(0)$. Bloodlevel ( $\_$), hepatosplenic uptake (_ — - . Data are expressed as mean values for groups of four rats.

Biodistribution of HPI:HPC: Chol liposomes in infected versus uninfected rats

The influence of $K$. pneumoniae lung infection on the blood circulation time and hepatosplenic uptake of HPI : HPC: Chol liposomes was determined by comparing the biodistribution in uninfected rats and infected rats at 3 days after bacterial inoculation of the left lung (Fig. 2). The patterns of hepatosplenic uptake and blood circulation of liposomes were similar in uninfected and infected rats. Only at $0.5 \mathrm{~h}$ after administration hepatosplenic uptake of liposomes was significantly higher in infected rats compared to uninfected rats $(P=0.001)$, and only at $5 \mathrm{~h}$ after administration the amount of liposomes circulating in the blood was significantly reduced in infected rats compared to uninfected rats $(P=0.007)$. Total recovery of liposomes from blood, liver, spleen, kidney and lung at $0.5 \mathrm{~h}$ after administration in infected rats was $92 \%$, being not significantly different from total recovery in uninfected rats.

Localization of HPI:HPC: Chol liposomes and $P G: P C$ : Chol liposomes in lung tissue

It was investigated whether the prolonged liposome blood circulation time of HPI:HPC: Chol liposomes was parallelled by enhanced localization of liposomes in infected left lung tissue. At $16 \mathrm{~h}$ after administration of $15 \mu \mathrm{mol}$ HPI : HPC: Chol liposomes per rat about $10 \%$ of the injected liposome dose was still circulating in the blood (Fig. 2). At that time localization of liposomes in lung tissue was determined in a group of 44 rats with varying intensity of infection. Bacterial numbers in the infected left lung ranged from $10^{5}$ up to $2 \cdot 10^{10}$, and weight of the infected left lung ranged from 0.45 up to $2.2 \mathrm{~g}$. In Fig. 3 it is shown that in rats with substantially increased bacterial numbers in the left lung the degree of localization of liposomes was up to 10-fold higher compared to the degree of localization of liposomes in the left lung of uninfected rats. Increased localization of liposomes appeared to be strongly correlated $(r=0.93, P<0.001)$ with the severity of infection manifested by the increase in weight of the infected left lung, as shown in Fig. 4. In the right lung of infected rats bacterial numbers ranged from 10 up to $10^{9}$. No development of an infectious process and parallel increase in lung weight was observed. Localization of liposomes in the right lung of infected rats was not increased compared to the localization in the right lung of uninfected rats (Fig. 3).

The localization of the relatively short circulating PG: PC: Chol liposomes in infected left lung tissue at $16 \mathrm{~h}$ after administration was determined in a group of 20 rats with varying intensity of infection and compared to the localization of long circulating HPI : HPC: Chol liposomes in infected left lung tissue of 20 rats with similar degrees of intensity of infection (left lung weights were in the range of 0.7 up to $2.0 \mathrm{~g}$ (Fig. 5). Lung localization of PG : PC: Chol liposomes 


\section{Localization of HPI:HPC:Chol liposomes}
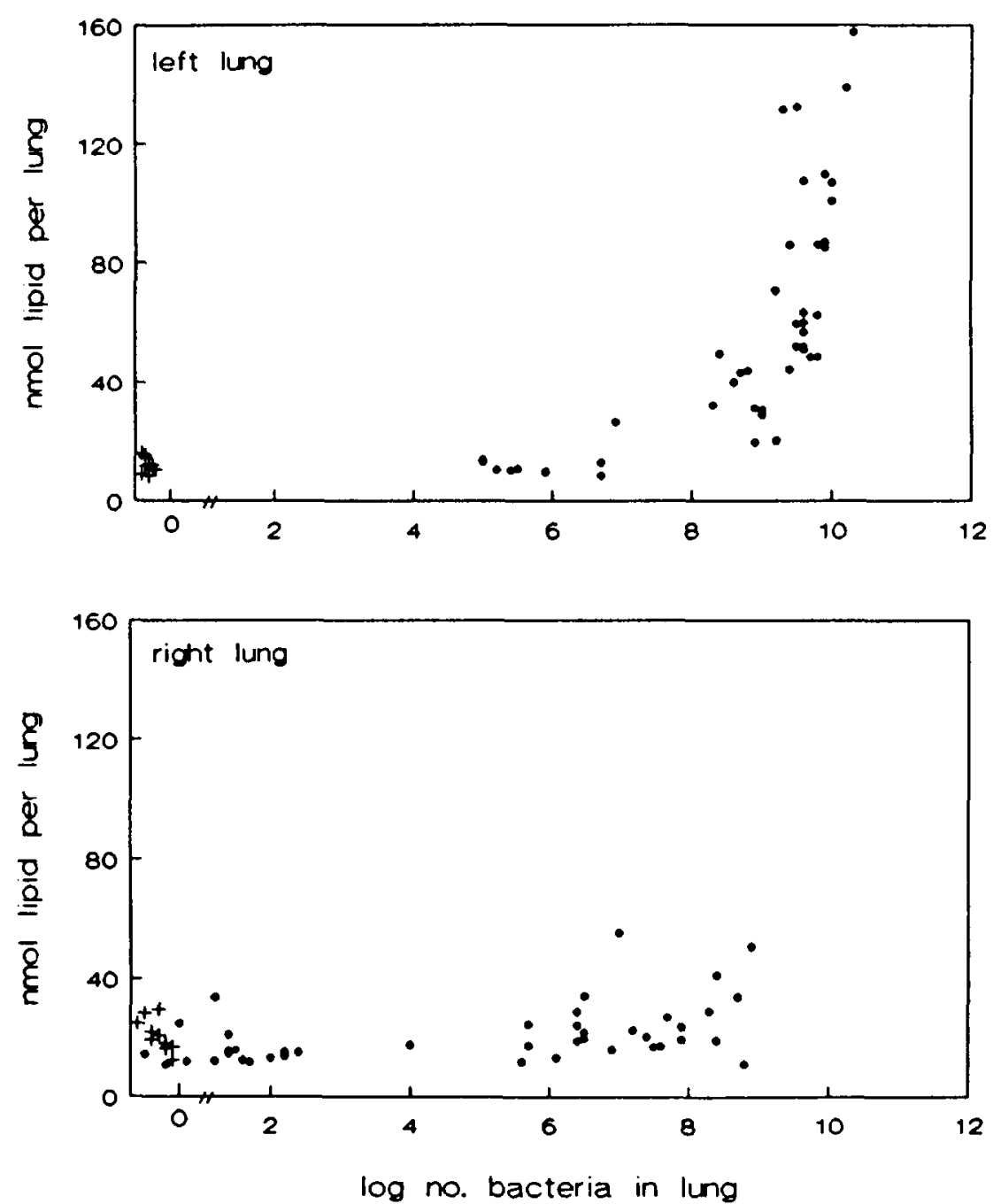

Fig. 3. Localization of ${ }^{67} \mathrm{Ga}-\mathrm{DF}$ labeled HPI:HPC:Chol (molar ratio, $1: 10: 5$ ) liposomes in the left lung and right lung of rats at $16 \mathrm{~h}$ after injection in relation to the bacterial numbers in the lung. A dose of $15 \mu$ mol liposomal lipid per rat was injected intravenously in a group of 44 rats with Klebsiella pneumoniae lung infection $(\bullet)$ or in a group of 10 uninfected rats $(+)$.

was significantly lower $(P=0.0008)$ compared to the localization of HPI : HPC: Chol liposomes.

Effect of liposomal lipid dose on the degree of localization of HPI: HPC: Chol liposomes in lung tissue of infected rats

HPI : HPC: Chol liposomes were administered to infected rats with increased left lung weights ranging from 0.7 up to $2.0 \mathrm{~g}$ at various doses ranging from 5 $\mu \mathrm{mol}$ up to $25 \mu \mathrm{mol}$ liposomal lipid per rat. Localization of liposomes in infected left lung tissue increased with increasing liposomal dose from $5 \mu \mathrm{mol}$ up to 15 $\mu \mathrm{mol}(r=0.83, P=0.006)$, whereas after administration of higher liposomal doses no further increase in localization was observed (Fig. 6). Also in the right lung a dose-related increase in localization of liposomes was observed. However, this increase was signifi- cantly less $(r=0.74, P=0.014)$ compared to that observed in the infected left lung.

\section{Discussion}

The development of long circulating liposome formulations has expanded considerably the potential therapeutic value of i.v. administered liposomes [8$12,18]$. These new types of liposomes have already been used in animals to deliver cytotoxic drugs more selectively to tumors and improve targeting of ligand-bearing liposomes to target cells within the vascular system $[9,19]$. In addition, it has been shown that encapsulation of the anticancer drug doxorubicin in long circulating liposomes results in remarkable alterations in pharmacokinetics and tissue distribution of the drug [9]. In the present study, such liposomes have been applied to 


\section{Localization of HPl:HPC:Chol liposomes}

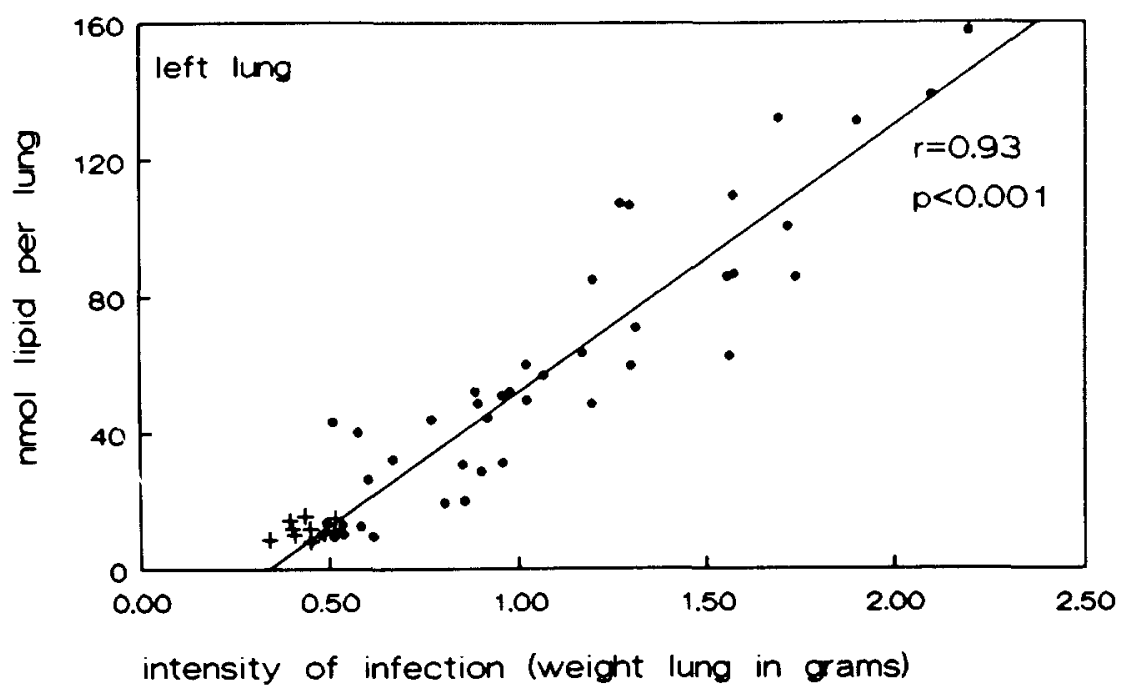

Fig. 4. Effect of the severity of infection on the localization of ${ }^{67} \mathrm{Ga}-\mathrm{DF}$ labeled HPI:HPC: Chol (molar ratio, $1: 10: 5$ ) liposomes in the left lung of rats at $16 \mathrm{~h}$ after injection. The increase in weight of the infected left lung was taken as measure of the intensity of the infection. A dose of 15 $\mu \mathrm{mol}$ liposomal lipid per rat was injected intravenously in a group of 44 rats with Klebsiella pneumoniae infection (•) or in a group of 10 uninfected rats $(+)$.

enhance the localization of liposomes in infected lung tissue.

In the past few years a number of studies were published in which several important characteristics of liposomes determining the biodistribution of liposomes after intravenous administration were identified [8$12,18]$. Factors contributing to prolongation of lipo- some circulation times and the stability of liposomes in blood are reduction of liposome size, and modifications of the bilayer composition yielding a negative charge, increased rigidity of the bilayer and increased hydrophilicity of the liposomal surface. The most favorable results were obtained with small sized liposomes containing cholesterol and solid-phase phospholipids such

\section{Localization of HPl:HPC:Chol and PG:PC:Chol liposomes}

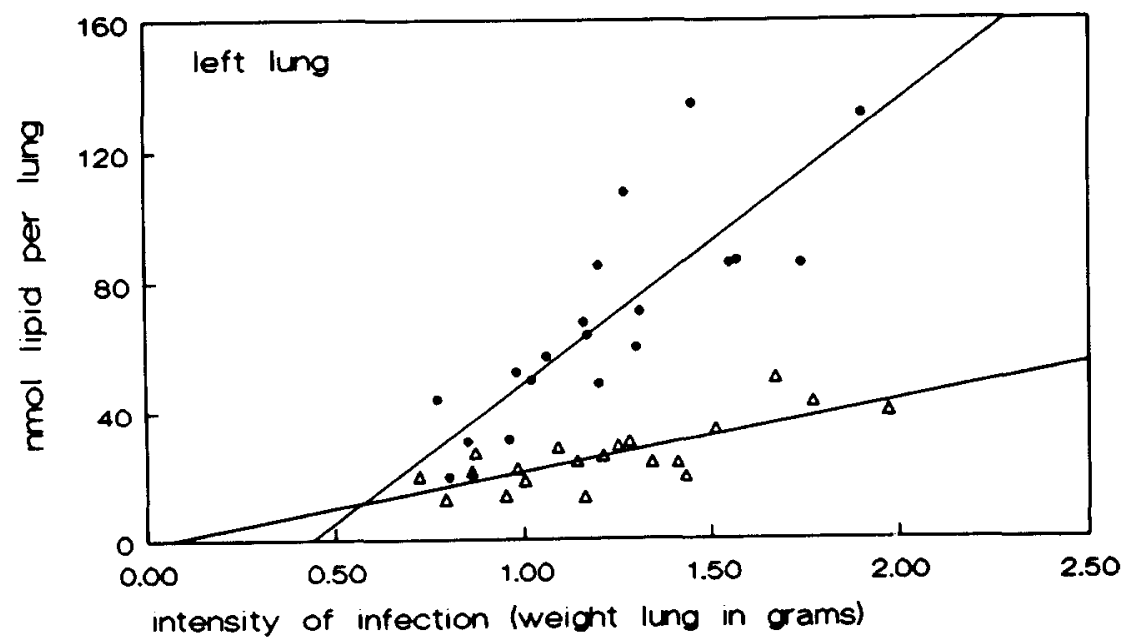

Fig. 5. Effect of the severity of infection on the localization of ${ }^{67} \mathrm{Ga}-\mathrm{DF}$ labeled liposomes in the left lung of rats at $16 \mathrm{~h}$ after injection. The increase in weight of the infected left lung was taken as measure of the intensity of the infection. HPI:HPC: Chol (molar ratio, 1:10:5) liposomes $(\bullet)$ and PG:PC:Chol (molar ratio, 1:10:5) liposomes $(\Delta)$ were injected intravenously into rats with Klebsiella pneumoniae lung infection at a dose of $15 \mu \mathrm{mol}$ lipid per rat. 


\section{Localization of HPl:HPC:Chol liposomes}

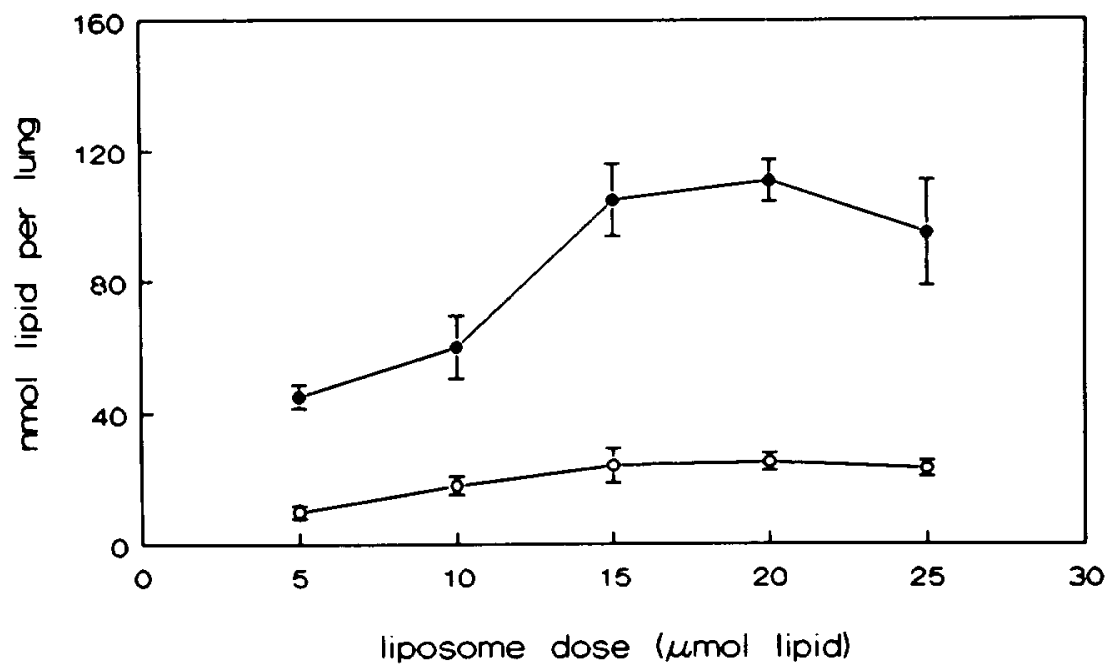

Fig. 6. Effect of liposomal lipid dose on degree of localization of ${ }^{67} \mathrm{Ga}-\mathrm{DF}$ labeled HPI:HPC: Chol (molar ratio, 1:10:5) liposomes in the left lung ( $\bullet$ and right lung (o) of rats at $16 \mathrm{~h}$ after injection. Liposomes were injected intravenously into rats with Klebsiella pneumoniae lung infection at various doses ranging from 5 up to $25 \mu \mathrm{mol}$ liposomal lipid per rat. Data are expressed as mean \pm S.E. values for groups of four rats.

as DSPC, in which the negatively charged monosialoganglioside GM-1 or HPI are included in the bilayer. In this study the potential of long circulating liposomes for enhanced drug delivery to sites of infection was addressed using an experimental pneumonia rat model. It was investigated whether intravenous administration of one of these liposome formulations with prolonged blood circulation time may result in significant localization of liposomes in infected lung tissue and whether the degree of localization is dependent on the intensity of infection. Inoculation of the left lung with a highly virulent $K$. pneumoniae strain resulted in acute infection in which the involvement of the lung tissue in the infectious process was reflected by a parallel increase in weight of the left lung. Biodistribution of liposomes was followed using a high-affinity ${ }^{67} \mathrm{Ga}$-DF complex as aqueous liposomal marker. Gabizon et al. showed that this complex is very appropriate for in vivo tracing of liposomes due to the advantages of minimal translocation of radioactive label to plasma proteins and the rapid renal clearance rate when the label is released from the liposomes extracellularly [15].

The two types of liposome used were relatively small and similar in size (mean particle diameter about 100 $\mathrm{nm}$ ), but differed in lipid composition, being HPI:HPC: Chol and PG:PC:Chol, respectively. For HPI : HPC: Chol liposomes with the same molar ratio of lipids a prolonged blood circulation time has been demonstrated in mice by Gabizon et al. $[8,10]$. They reported that $9 \%$ of the injected liposome dose was recovered from the blood at $24 \mathrm{~h}$ after administration. We were able to confirm their findings in our experimental study in rats. The HPI:HPC: Chol liposomes showed relatively long blood circulation time: at 10,16 and $24 \mathrm{~h}$ after administration, 23, 10 and $4 \%$ of the injected dose, respectively, was still circulating in the bloodstream. However, changing the lipid composition into PG: PC: Chol while maintaining a similar size resulted in a significant decrease in blood circulation time. The observations with the two types of liposome suggest that prolonged liposome circulation correlates with reduced hepatosplenic uptake.

Comparison of the behavior of long circulating liposomes in uninfected and infected rats revealed that the bacterial lung infection did not affect the biodistribution markedly. The slightly increased hepatosplenic uptake of liposomes observed at $0.5 \mathrm{~h}$ after injection may be explained by an increased uptake of liposomes by liver and spleen macrophages being activated by bacteria circulating in the blood at this stage of lung infection. Importantly, however, biodistribution with respect to lung uptake was clearly modified. It was observed that the long circulating HPI: HPC: Chol liposomes showed significant localization in infected left lung tissue. At $16 \mathrm{~h}$ after administration when about $10 \%$ of injected liposomes was still present in the bloodstream, localization of liposomes in the infected left lung was increased up to 10 -fold compared to the left lung of uninfected rats. The increased degree of localization of liposomes in the infected left lung is likely to be related to the local infectious process, because in the right lung of the same animal in which bacteria were present without signs of development of an infectious process, the degree of localization of liposomes was not increased compared to the degree of localization observed in uninfected rats. Interestingly, 
the increased localization of liposomes in the infected left lung appeared to be highly correlated with the intensity of the infection, the latter being reflected by the parallel rise in weight of the infected left lung. Compared to HPI:HPC: Chol liposomes localization of PG: $\mathrm{PC}$ : Chol liposomes in the infected left lung was significantly lower. This suggests that the degree of localization of liposomes in the infected tissue is dependent on the residence time of liposomes in the blood compartment. Prolonged circulation would enable the liposomes to extravasate more efficiently. Extravasation of liposomes at the site of infection is probably related to locally increased capillary permeability or damaged endothelium secondary to the infectious process. Besides the long residence time of liposomes in the blood compartment, phagocytosis of liposomes by inflammatory cells infiltrating into the infected tissue may also acount for enhanced localization of liposomes in the infected lung. The role of inflammatory cells in this respect has to be elucidated.

It appeared that the extent of localization of liposomes in infected tissue is dependent on the liposomal dose administered, saturating around a dose of 15 $\mu \mathrm{mol}$ lipid/rat. This observation suggests that enhanced localization of liposomes in infected tissue is not only the result of extravasation of liposomes at the site of infection through leaky or damaged endothelium, since in that case a saturation effect is not expected. The result indicates perhaps cellular and/or protein involvement.

In summary the experiments presented here indicate that liposomes with prolonged blood circulation time achieve increased localization in infected lung tissue compared to conventional (short circulating) liposomes. Lung localization of liposomes is dependent on the intensity of infection, is significantly increased from the moment that bacterial multiplication in the lung results in a local inflammatory response, and appeared to be correlated with increased involvement of the lung tissue in the infectious process reflected by a rise in weight of the infected tissuc. Whether liposomes with prolonged blood circulation time can bc used for improved delivery of antibioties to the site of infection is the subject of current investigations.

\section{References}

1 Bakker-Woudenberg, I.A.J.M. and Lokerse, A.F. (1991) Scand. J. Infect. Dis. Suppl. 74, 34-41.

2 Swenson, C.E., Popescu, M.C. and Ginsberg. R.S. (1988) CRC Crit. Rev. Microbiol. 15 (Suppl. 1), 1-31.

3 Emmen, F. and Storm. G. (1987) Pharm. Weekbl. (Sci) 9, 162-171. 4 Coune, A. (1988) Infection 16, 141-147.

5 Bakker-Woudenberg, I.A.J.M.. Roerdink. F.H. and Scherphof. G.L. (1988) in Liposomes as drug carriers. Trends and progress (Gregoriadis, G., ed.), pp. 325-336, John Wiley and Sons, Chichester.

6 Juliano, R.L. (1989) in Drug carrier systems (Roerdink, F.H.D. and Kroon, A.M., eds.), pp. 249-279, John Wiley and Sons. Chichester.

7 Bakker-Woudenberg, I.A.J.M., Lokerse, A.F. and Roerdink, F.H. (1989) J. Pharmacol. Exp. Ther. 251, 321-327.

8 Gabizon, A. and Papahadjopoulos, D. (1988) Proc. Natl. Acad. Sci. USA $85,6949-6953$.

9 Gabizon, A., Shiota, R. and Papahadjopoulos, D. (1989) J. Natl Cancer Inst. 81, 1484-1488.

10 Gabizon, A., Price, D.C., Huberty, J., Bresalier, R.S. and Papahadjopoulos, D. (1990) Cancer Res. 50, 6371-6378.

11 Allen, T.M. and Chonn, A. (1987) FEBS Lett. 223, 42-46.

12 Allen, T.M., Hansen, C. and Rutledge, J. (1989) Biochim. Biophys. Acta 981, 27-35.

13 Bakker-Woudenberg, I.A.J.M., Lokerse, A.F., Roerdink, F.H. Regts, D. and Michel, M.F. (1985) J. Infect. Dis. 151, 917-924.

14 Ames, B.N. and Dubin, D.T. (1960) J. Biol. Chem. 235, 769-775.

15 Gabizon, A., Huberty, J., Straubinger, R.M., Price, D.C. and Papahadjopoulos, D. (1988) J. Liposome Res. 1, 123-135.

16 Bakker-Woudenberg, I.A.J.M., Van den Berg, J.C. and Michel. M.F. (1982) Antimicrob. Agent Chemother. 22, 1042-1050.

17 Heaton, W.A., Davis, H.H., Welch, M.J., Mathias, C.J., Joist. J.H., Sherman, L.A. and Siegel, B.A. (1979) Br. J. Haematol. 42. $613-622$.

18 Hwang, K.J., Padki, M.M., Chow, D.D., Essien, H.E., Lai, J.Y. and Beaumier, P.L. (1987) Biochim. Biophys. Acta 910, 88-96.

19 Maruyama, K., Kennel, S.J. and Huang. L. (1990) Proc. Natl Acad. Sci. USA 87, 5744-5748. 